OPEN ACCESS

Edited by:

Zhendong Jin,

The University of lowa, United States

Reviewed by:

Yingxia $L i$

Fudan University, China

Hitendra M. Patel,

Sardar Patel University, India

*Correspondence:

Toshio Morikawa

morikawa@kindai.ac.jp

Specialty section

This article was submitted to

Organic Chemistry,

a section of the journal

Frontiers in Chemistry

Received: 24 December 2019 Accepted: 04 March 2020

Published: 20 March 2020

Citation:

Morikawa T, Luo F, Manse Y, Sugita H,

Saeki S, Chaipech S,

Pongpiriyadacha $Y$, Muraoka $O$ and

Ninomiya K (2020) Geranylated

Coumarins From Thai Medicinal Plant

Mammea siamensis With

Testosterone $5 \alpha$-Reductase Inhibitory

Activity. Front. Chem. 8:199.

doi: 10.3389/fchem.2020.00199

\section{Geranylated Coumarins From Thai Medicinal Plant Mammea siamensis With Testosterone $5 \alpha$-Reductase Inhibitory Activity}

\author{
Toshio Morikawa ${ }^{1 *}$, Fenglin Luo ${ }^{1}$, Yoshiaki Manse ${ }^{1}$, Hidemi Sugita ${ }^{1}$, Shunsuke Saeki ${ }^{1}$, \\ Saowanee Chaipech ${ }^{1,2}$, Yutana Pongpiriyadacha ${ }^{3}$, Osamu Muraoka ${ }^{1}$ and \\ Kiyofumi Ninomiya ${ }^{1}$
}

${ }^{1}$ Pharmaceutical Research and Technology Institute, Kindai University, Osaka, Japan, ${ }^{2}$ Faculty of Agro-Industry, Rajamangala University of Technology Srivijaya, Nakhon Si Thammarat, Thailand, ${ }^{3}$ Faculty of Science and Technology, Rajamangala University of Technology Srivijaya, Nakhon Si Thammarat, Thailand

Geranylated coumarin constituents, kayeassamin I (1) and mammeasins E (2) and F (3) were newly isolated from the methanol extract of the flowers of Mammea siamensis (Calophyllaceae) originating in Thailand, along with five known isolates, such as mammea E/BC (23), deacetylmammea E/AA cyclo D (31), deacetylmammea E/BB cyclo D (32), mammea AVAA cyclo $F(\mathbf{3 4})$, and mammea AVAC cyclo $F(\mathbf{3 5})$. These compounds (1-3) were obtained as an inseparable mixture (ca. 1:1 ratio) of the $3^{\prime \prime} R$ and $3^{\prime \prime} S$ forms, respectively. Among the isolated coumarins from the extract, mammeasins $E$ $(\mathbf{2}, 22.6 \mu \mathrm{M}), \mathrm{A}(\mathbf{4}, 19.0 \mu \mathrm{M})$, and $\mathrm{B}(\mathbf{5}, 24.0 \mu \mathrm{M})$, kayeassamins $\mathrm{E}(\mathbf{9}, 33.8 \mu \mathrm{M}), \mathrm{F}(\mathbf{1 0}$, $15.9 \mu \mathrm{M})$, and $\mathrm{G}(\mathbf{1 1}, 17.7 \mu \mathrm{M})$, surangin $\mathrm{C}(\mathbf{1 3}, 5.9 \mu \mathrm{M})$, and mammeas $\mathrm{A} / \mathrm{AA}(\mathbf{1 7}$, $19.5 \mu \mathrm{M})$, E/BB $(\mathbf{2 2}, 16.8 \mu \mathrm{M})$, and A/AA cyclo $\mathrm{F}(\mathbf{3 4}, 23.6 \mu \mathrm{M})$, were found to inhibit testosterone $5 \alpha$-reductase.

Keywords: Mammea siamensis, mammeasin, $5 \alpha$-reductase inhibitor, geranylated coumarin, calophyllaceae

\section{INTRODUCTION}

The Calophyllaceae plant Mammea siamensis (Miq.) T. Anders. is a small evergreen tree distributed in Thailand (locally called "Sarapi" or "Saraphi"), Laos, Cambodia, Vietnam, and Myanmar. The flowers of this plant have traditionally been used as a heart tonic, fever-lowering, and enhancement of appetite in Thailand (Morikawa et al., 2012; Tung et al., 2013; Ninomiya et al., 2016; Sangkaruk et al., 2017). Previous chemical studies on the flowers (Kaweetripob et al., 2000; Prachyawarakorn et al., 2000, 2006a; Mahidol et al., 2002; Morikawa et al., 2012; Ninomiya et al., 2016), seeds (Laphookhieo et al., 2006, 2007), twigs (Poobrasert et al., 1998; Prachyawarakorn et al., 2006a,b), and bark (Ngo et al., 2010) of M. siamensis reported on the isolation of several coumarins and xanthones, etc. With regard to the biological studies on M. siamensis and its constituents, cytotoxicity, antiproliferative, and apoptotic effects against several tumor and cancer cell lines (Ngo et al., 2010; Tung et al., 2013; Noysang et al., 2014; Uto et al., 2016; Sangkaruk et al., 2017), suppressive effects on inducible nitric oxide synthase expression in RAW264.7 cells (Morikawa et al., 2012), and aromatase inhibitory activity (Ninomiya et al., 2016; Tanabe et al., 2017) have been reported. Further separation of the constituents in the extract resulted in the isolation of three geranylated coumarins, kayeassamin I (1) and mammeasins E (2) and F (3). Here, we conducted the isolation and structural verification of $\mathbf{1 - 3}$, as well as examined the testosterone $5 \alpha$-reductase 
inhibitory activity of its coumarin constituents (1-35), including five new isolates, such as mammea $\mathrm{E} / \mathrm{BC}$ (23), deacetylmammea E/AA cyclo D (31), deacetylmammea E/BB cyclo D (32), mammea A/AA cyclo F (34), and mammea A/AC cyclo F (35).

\section{MATERIALS AND METHODS}

\section{General Experimental Procedures}

The following instruments were used to obtain physical data: a SEPA-300 digital polarimeter (Horiba Ltd., Kyoto, Japan, $l=5 \mathrm{~cm}$ ) for specific rotations; an UV-1600 spectrometer (Shimadzu Co., Kyoto, Japan) to record UV spectra; a FTIR-8100 spectrometer (Shimadzu Co.) to measure IR spectra; a JNMECA800 (800 MHz), JNM-ECA700 (700 MHz), JNM-ECA500 (500 MHz), and JNM-ECS400 and JNM-AL400 (400 MHz) spectrometers (JEOL Ltd., Tokyo, Japan) to determine ${ }^{1} \mathrm{H}$ NMR spectra; JNM-ECA800 (200 MHz), JNM-ECA700 (175 MHz), JNM-ECA500 (125 MHz), and JNM-ECS400 and JNM-AL-400 $\left(100 \mathrm{MHz}\right.$ ) spectrometers (JEOL Ltd.) to record ${ }^{13} \mathrm{C}$ NMR spectra in $\mathrm{CDCl}_{3}$ at room temperature $\left(25^{\circ} \mathrm{C}\right)$ with tetramethylsilane as an internal standard; an Exactive Plus Orbitrap mass spectrometer (Thermo Fisher Scientific Inc., Waltham, MA, USA) to measure ESIMS and HRESIMS; an HPLC detector, SPD-10A vp UV-Vis (Shimadzu Co.); and Cosmosil $5 \mathrm{C}_{18}$-MSII (Nacalai Tesque, Inc., Kyoto, Japan) HPLC columns (4.6 mm i.d. $\times 250 \mathrm{~mm}$ and $20 \mathrm{~mm}$ i.d. $\times 250 \mathrm{~mm}$ ) for analytical and preparative purposes, respectively.

The following materials and experimental conditions were used for the column chromatography (CC): normal-phase silica gel CC, silica gel $60 \mathrm{~N}$ (Kanto Chemical Co., Ltd., Tokyo, Japan; 63-210 mesh, spherical, neutral); reversed-phase ODS CC, Chromatorex ODS DM1020T (Fuji Silysia Chemical, Ltd., Aichi, Japan; 100-200 mesh); TLC, pre-coated TLC plates with silica gel $60 \mathrm{~F}_{254}$ (Merck, Darmstadt, Germany, $0.25 \mathrm{~mm}$, normalphase) and silica gel RP-18 $\mathrm{WF}_{254 \mathrm{~S}}$ (Merck, Darmstadt, Germany, $0.25 \mathrm{~mm}$, reversed-phase); reversed-phase HPTLC, pre-coated TLC plates with silica gel RP-18 $\mathrm{WF}_{254 \mathrm{~S}}$ (Merck, $0.25 \mathrm{~mm}$ ); detection was performed by spraying $1 \% \mathrm{Ce}\left(\mathrm{SO}_{4}\right)_{2}-10 \%$ aqueous $\mathrm{H}_{2} \mathrm{SO}_{4}$, followed by heating.

\section{Plant Material}

The flowers of Mammea siamensis were collected from the Nakhonsithammarat Province, Thailand, in September 2006, as described previously (Morikawa et al., 2012; Ninomiya et al., 2016). The plant material was identified by one of the authors (Y. P.). A voucher specimen (2006.09. Raj-04) for this plant has been deposited in our laboratory.

\section{Extraction and Isolation}

Dried flowers of $M$. siamensis $(1.8 \mathrm{~kg})$ were extracted three times with $\mathrm{MeOH}$ under reflux for $3 \mathrm{~h}$. Evaporation of the combined extracts under reduced pressure afforded the $\mathrm{MeOH}$ extract $(463.7 \mathrm{~g}, 25.66 \%)$. An aliquot $(413.7 \mathrm{~g})$ of the extract was partitioned into an EtOAc- $\mathrm{H}_{2} \mathrm{O}(1: 1, \mathrm{v} / \mathrm{v})$ mixture to furnish an EtOAc-soluble fraction $(110.34 \mathrm{~g}, 6.84 \%)$ and an aqueous phase. An aliquot $(89.45 \mathrm{~g})$ of the EtOAc-soluble fraction was subjected to normal-phase silica gel CC $[3.0 \mathrm{~kg}, n$-hexane-EtOAc
$(10: 1 \rightarrow 7: 1 \rightarrow 5: 1, \mathrm{v} / \mathrm{v}) \rightarrow \quad$ EtOAc $\rightarrow \quad \mathrm{MeOH}]$ to give 11 fractions [Fr. 1 (3.05 g), Fr. 2 (2.86 g), Fr. 3 (11.71 g), Fr. 4 (1.62 g), Fr. 5 (4.15 g), Fr. 6 (6.29 g), Fr. 7 (2.21 g), Fr. 8 (2.94 g), Fr. 9 (10.23 g), Fr. 10 (11.17 g), and Fr. 11 (21.35 g)]. Fraction $5(4.15 \mathrm{~g})$ was subjected to reversed-phase silica gel CC [120 g, $\mathrm{MeOH}-\mathrm{H}_{2} \mathrm{O}(80: 20 \rightarrow 85: 15, \mathrm{v} / \mathrm{v}) \rightarrow \mathrm{MeOH} \rightarrow$ acetone $]$ to afford six fractions [Fr. 5-1 (115.7 mg), Fr. 5-2 (2789.8 mg), Fr. 5-3 (515.4 mg), Fr. 5-4 (430.0 mg), Fr. 5-5 (119.2 mg), and Fr. 5-6 (110.0 mg)] as reported previously (Ninomiya et al., 2016). Fraction 5-2 (517.0 mg) was purified by HPLC [Cosmosil $5 \mathrm{C}_{18}$-MS-II, $\mathrm{MeOH}-1 \%$ aqueous $\left.\mathrm{AcOH}(85: 15, \mathrm{v} / \mathrm{v})\right]$ to give mammea A/AC cyclo F (35, 4.6 mg, 0.0019\%) (Morel et al., 1999; Prachyawarakorn et al., 2000; Guilet et al., 2001) together with mammeas A/AA (17, $101.2 \mathrm{mg}, 0.0418 \%)$, A/AC (19, $112.9 \mathrm{mg}$, $0.0466 \%)$, A/AA cyclo D $(24,2.7 \mathrm{mg}, 0.0011 \%), \mathrm{E} / \mathrm{BC}$ cyclo D (29, $14.0 \mathrm{mg}, 0.0058 \%)$, and E/BD cyclo D (30, $1.8 \mathrm{mg}, 0.0015 \%)$ (Mahidol et al., 2002). Fraction 5-3 (515.4 mg) was purified by HPLC [Cosmosil $5 \mathrm{C}_{18}$-MS-II, $\mathrm{MeOH}-1 \%$ aqueous $\mathrm{AcOH}$ $(85: 15, \mathrm{v} / \mathrm{v})]$ to give mammea A/AA cyclo $\mathrm{F}$ (34, $13.2 \mathrm{mg}$, $0.0010 \%$ ) (Prachyawarakorn et al., 2000; Guilet et al., 2001) together with 19 (45.6 mg, 0.0035\%), 24 (14.9 mg, 0.0011\%), mammeas A/AB cyclo D (25, $46.4 \mathrm{mg}, 0.0035 \%)$ and A/AC cyclo $\mathrm{D}(26,30.1 \mathrm{mg}, 0.0023 \%)$. Fraction $6(6.29 \mathrm{~g})$ was subjected to reversed-phase silica gel CC $\left[200 \mathrm{~g}, \mathrm{MeOH}-\mathrm{H}_{2} \mathrm{O}(80: 20 \rightarrow\right.$ $90: 10 \rightarrow 95: 5, \mathrm{v} / \mathrm{v}) \rightarrow \mathrm{MeOH} \rightarrow$ acetone] to afford 10 fractions [Fr. 6-1 (44.7 mg), Fr. 6-2 (157.2 mg), Fr. 6-3 (928.8 mg), Fr. 6-4 (3117.0 mg), Fr. 6-5 (128.8 mg), Fr. 6-6 (487.1 mg), Fr. 6-7 (230.8 mg), Fr. 6-8 (280.5 mg), Fr. 6-9 (102.9 mg), and Fr. 6-10 (96.5 mg)] as reported previously (Morikawa et al., 2012; Ninomiya et al., 2016). Fraction 6-4 (536.2 mg) was purified by HPLC [Cosmosil $5 \mathrm{C}_{18}$-MS-II, $\mathrm{MeOH}-1 \%$ aqueous $\mathrm{AcOH}$ $(90: 10, \mathrm{v} / \mathrm{v})]$ to give kayeassamin I $(1,7.2 \mathrm{mg}, 0.0032 \%)$ (Win et al., 2008b), mammeasin E (2, $16.5 \mathrm{mg}, 0.0073 \%)$, and 35 $(11.0 \mathrm{mg}, 0.0049 \%)$ together with mammeasins $\mathrm{A}(4,65.8 \mathrm{mg}$, $0.0293 \%)$ and B (5, 21.6 mg, 0.0096\%), surangin B (12, $58.2 \mathrm{mg}$, $0.0259 \%), 17$ (17.0 mg, 0.0076\%), mammea A/AB (18, $10.7 \mathrm{mg}$, $0.0048 \%)$, and $19(112.6 \mathrm{mg}, 0.0501 \%)$. Fraction 7 (2.21 g) was subjected to reversed-phase ODS CC [47.0 g, MeOH- $\mathrm{H}_{2} \mathrm{O}(60: 40$ $\rightarrow \quad 80: 20 \rightarrow 90: 10, \mathrm{v} / \mathrm{v}) \rightarrow \mathrm{MeOH} \rightarrow$ acetone] to afford five fractions [Fr. 7-1 (187.3 mg), Fr. 7-2 (912.0 mg), Fr. 7-3 (275.2 mg), Fr. 7-4 (30.0 mg), and Fr. 7-5 (44.0 mg)]. Fraction 7-2 $(912.0 \mathrm{mg})$ was purified by HPLC [column: Cosmosil $5 \mathrm{C}_{18}-\mathrm{MS}-$ II, detection: UV $(230 \mathrm{~nm})$, mobile phase: $\mathrm{MeOH}-1 \%$ aqueous $\left.\mathrm{H}_{2} \mathrm{O}(85: 15, \mathrm{v} / \mathrm{v})\right]$ to give mammeasin $\mathrm{E} / \mathrm{BC}$ (23, $99.0 \mathrm{mg}$, 0.0076\%) (Yang et al., 2005). Fraction 7-3 (275.2 mg) was purified by HPLC [Cosmosil 5C 18 -MS-II, UV (230 nm), MeOH$1 \%$ aqueous $\mathrm{AcOH} 85: 15, \mathrm{v} / \mathrm{v}$ ] to give 1 (52.1 mg, 0.0040\%), 2 (34.1 $\mathrm{mg}, 0.0026 \%)$, and mammeasin F (3, $19.5 \mathrm{mg}, 0.0015 \%)$. Fraction $9(10.23 \mathrm{~g})$ was subjected to reversed-phase silica gel $\mathrm{CC}\left[300 \mathrm{~g}, \mathrm{MeOH}-\mathrm{H}_{2} \mathrm{O}(80: 20 \rightarrow 90: 10, \mathrm{v} / \mathrm{v}) \rightarrow \mathrm{MeOH}\right.$ $\rightarrow$ acetone] to afford five fractions [Fr. 9-1 (2809.0 mg), Fr. 9-2 (5678.0 mg), Fr. 9-3 (385.9 mg), Fr. 9-4 (422.0 mg), and Fr. 9-5 (51.9 mg)] as reported previously (Morikawa et al., 2012; Ninomiya et al., 2016). Fraction 9-1 (544.5 mg) was purified by HPLC [Cosmosil $5 \mathrm{C}_{18}$-MS-II, $\mathrm{MeOH}-1 \%$ aqueous $\mathrm{AcOH}$ $(85: 15, \mathrm{v} / \mathrm{v})]$ to give deacetylmammeas E/AA cyclo D (31, $1.3 \mathrm{mg}$, 0.0005\%) (Mahidol et al., 2007) and E/BB cyclo D (32, $6.1 \mathrm{mg}$, 
0.0023\%) (Mahidol et al., 2007) together with kayeassamins E (9, 28.6 mg, 0.0113\%), F (10, $98.7 \mathrm{mg}, 0.0390 \%)$, and G (11, $43.4 \mathrm{mg}, 0.0171 \%)$, deacetylmammea E/BC cyclo D (33, $18.6 \mathrm{mg}$, $0.0073 \%)$, and benzoic acid (10.9 mg, $0.0043 \%)$.

\section{Kayeassamin I (1)}

Pale yellow oil; $[\alpha]_{\mathrm{D}}^{25}-50.4\left(c 0.63, \mathrm{CHCl}_{3}\right)\left\{[\alpha]_{\mathrm{D}}^{23}-35.52(c 0.90\right.$, $\mathrm{CHCl}_{3}$ ) (Win et al., 2008a) $\} ;{ }^{1} \mathrm{H}$ and ${ }^{13} \mathrm{C}$ NMR spectroscopic data (see Table 1); Negative-ion ESIMS $m / z 439[\mathrm{M}-\mathrm{H}]^{-}$; HRESIMS $\mathrm{m} / z 439.2116$ (calcd for $\mathrm{C}_{26} \mathrm{H}_{31} \mathrm{O}_{6}, 439.2115$ ) (Figures S3-S7).

\section{Mammeasin E (2)}

Pale yellow oil; $[\alpha]_{\mathrm{D}}^{25}-58.9\left(\right.$ c $\left.0.12, \mathrm{CHCl}_{3}\right)$; UV $(\mathrm{MeOH}) \lambda_{\max }$ $\mathrm{nm}(\log \varepsilon)$ : 223 (4.01), 278 (4.12), 302 (4.12); IR (KBr) $v_{\max }$ $\mathrm{cm}^{-1}: 1,740,1,713,1,613,1,454,1,408,1,284,1,126,1,049 ;{ }^{1} \mathrm{H}$ and ${ }^{13} \mathrm{C}$ NMR spectroscopic data (see Table 2); Negative-ion ESIMS $m / z 453[\mathrm{M}-\mathrm{H}]^{-}$; HRESIMS $m / z 453.2272$ (calcd for $\mathrm{C}_{27} \mathrm{H}_{33} \mathrm{O}_{6}, 453.2272$ ) (Figures S8-S12).

\section{Mammeasin F (3)}

Pale yellow oil; $[\alpha]_{\mathrm{D}}^{25}-42.1\left(\right.$ c 0.45, $\left.\mathrm{CHCl}_{3}\right)$; UV $(\mathrm{MeOH}) \lambda_{\max }$ $\mathrm{nm}(\log \varepsilon): 224$ (3.89), 298 (3.82); IR (KBr) $v_{\max } \mathrm{cm}^{-1}: 1,732$, $1,713,1,605,1,454,1,381,1,261,1,126,1,049 ;{ }^{1} \mathrm{H}$ and ${ }^{13} \mathrm{C}$ NMR spectroscopic data (see Table 2); Negative-ion ESIMS $m / z 453$ [M - $\mathrm{H}]^{-}$; HRESIMS $m / z 453.2287$ (calcd for $\mathrm{C}_{27} \mathrm{H}_{33} \mathrm{O}_{6}, 453.2272$ ) (Figures S13-S17).

\section{DDQ Oxidation of Kayeassamin A (8) and Surangins C (13) and D (14)}

A solution of kayeassamin A $(\mathbf{8}, 9.0 \mathrm{mg})$ in dry-toluene $(2.0 \mathrm{~mL})$ was treated with 2,3-dichloro-5,6-dicyano-p-benzoquinone (DDQ, $10.0 \mathrm{mg}$ ) and the solution stirred at room temperature $\left(25^{\circ} \mathrm{C}\right)$ for $4 \mathrm{~h}$. The aqueous solution was saturated with sodium hydrogen carbonate $\left(\mathrm{NaHCO}_{3}\right)$ and extracted with EtOAc. The EtOAc extract was washed with brine then dried over anhydrous magnesium sulfate $\left(\mathrm{MgSO}_{4}\right)$ and filtered. Removal of the solvent under reduced pressure gave a residue, which was purified by HPLC [Cosmosil $5 \mathrm{C}_{18}$-MS-II, $\mathrm{MeOH}-1 \%$ aqueous $\mathrm{AcOH}$ $(85: 15, \mathrm{v} / \mathrm{v})]$ to give kayeassamin I $(1,3.8 \mathrm{mg}, 46 \%)$. Through the similar procedure, mammeasin $\mathrm{E}(2,3.3 \mathrm{mg}, 38 \%)$ and mammeasin F (3, $2.0 \mathrm{mg}, 17 \%)$ were obtained from surangins D $(14,9.6 \mathrm{mg})$ and $\mathrm{C}(13,12.7 \mathrm{mg})$, respectively.

\section{Assay for Testosterone $5 \alpha$-Reductase Inhibitory Activity}

The experiment was performed in accordance with previously reported methods (Matsuda et al., 2001; Lee et al., 2012; Koseki et al., 2015) with slight modifications. In brief, the assay was performed in 48-well microplates (Sumitomo Bakelite Co., Ltd., Tokyo, Japan). The reaction solution was pre-incubated with or without a test sample ( $5 \mu \mathrm{L} /$ well, dissolved in DMSO), in a potassium phosphate buffer $(40 \mathrm{mM}, \mathrm{pH} 6.5,490 \mu \mathrm{L} /$ well $)$ containing substrate $(0.35 \mathrm{nmol}$ of testosterone, Tokyo Chemical Industry Co., Ltd., Tokyo, Japan) and NADPH (10 nmol, Oriental Yeast Co., Ltd., Tokyo, Japan) at room temperature $\left(25^{\circ} \mathrm{C}\right)$ for $20 \mathrm{~min}$. The enzymatic reaction was initiated by the addition of rat liver $\mathrm{S} 9$ fractions $(10 \mu \mathrm{L} /$ well, dissolved in the phosphate buffer, $20.6 \mu \mathrm{g} /$ well, Oriental Yeast Co., Ltd., Tokyo, Japan, lot no. 109031513) at $37^{\circ} \mathrm{C}$ for $30 \mathrm{~min}$. After incubation, the reaction mixture was immediately heated in boiling water for $2 \mathrm{~min}$ to stop the reaction. Then the reaction solution of each well was transferred to a microtube and extracted with $500 \mu \mathrm{L}$ of EtOAc. After the microtube was centrifuged $(10,000 \mathrm{rpm}, 5 \mathrm{~min})$, an aliquot of each EtOAc phase $(300 \mu \mathrm{L})$ was transferred into another tube. The solvent in the tube was evaporated and the residue was dissolved in $30 \mu \mathrm{L}$ of acetonitrile containing an internal standard (I.S.) fludrocortisone acetate $(20 \mu \mathrm{g} / \mathrm{mL}$, Sigma-Aldrich, Co., LLC, St. Louis, USA). An aliquot of $2 \mu \mathrm{L}$ was injected into the HPLC under the following conditions [Instrument: a series LC-20A Prominence HPLC system (Shimadzu Co., Kyoto, Japan); Detection: UV (254 nm); Column: Cosmosil 5C 18 -MS-II (Nakalai Tesque Inc., Kyoto, Japan, $5 \mu \mathrm{m}$ particle size, $2.0 \mathrm{~mm}$ i.d. $\times 150 \mathrm{~mm}$ ); Column temperature: $40^{\circ} \mathrm{C}$; Mobile phase: $\mathrm{MeOH}-\mathrm{H}_{2} \mathrm{O}(60: 40$, v/v); Flow rate: $0.2 \mathrm{~mL} / \mathrm{min}$; retention time: $13.5 \mathrm{~min}$ for testosterone and $8.0 \mathrm{~min}$ for I.S. A similar procedure that described above was carried out for the control tubes. The $5 \alpha$-reductase inhibitory activity was determined from the following equation using the peak area ratios ( $r=$ testosterone/I.S.). Experiments were performed in triplicate or quadruple, and $\mathrm{IC}_{50}$ values were determined graphically. The $5 \alpha$-reductase inhibitor finasteride (Tokyo Chemical Industry Co., Ltd., Tokyo, Japan) was used as a reference compound.

$$
\text { Inhibition }(\%)=[r(\mathrm{~T})-r(\mathrm{C}) / r(\mathrm{~B})-r(\mathrm{C})] \times 100
$$

Control (C): enzyme $(+)$, test sample $(-)$; Test $(\mathrm{T})$ : enzyme $(+)$, test sample $(+)$; Blank $(B)$ : enzyme $(-)$, test sample $(+)$.

\section{Statistics}

Values are expressed as mean \pm S.E.M. One-way analysis of variance (ANOVA), followed by Dunnett's test, was used for statistical analysis. Probability $(p)$ values $<0.05$ were considered significant.

\section{RESULTS AND DISCUSSION}

\section{Effects of the Methanol Extract From the Flowers of $M$. siamensis on Testosterone $5 \alpha$-Reductase}

The male sex hormones, androgens, play a crucial role in the development, growth and function of the prostate, and other androgen-sensitive peripheral tissues. In the prostate gland, androgens are involved in benign prostatic hyperplasia and prostate cancer, as well as in skin disorders, such as acne, seborrhea, androgenic alopecia, and hirsutism. Among the androgens, testosterone is the most abundant in serum and secreted primarily by the testicles and ovaries. The enzyme steroid $5 \alpha$-reductase catalyzes the conversion of testosterone to the most potent natural androgen, $5 \alpha$-dihydrotestosterone (Yamana et al., 2010; Yao et al., 2011; Azzouni et al., 2012). Therefore, inhibition of testosterone $5 \alpha$-reductase could be useful for the treatment of the above diseases. To date, three types 
TABLE 1 | ${ }^{1} \mathrm{H}$ and ${ }^{13} \mathrm{C}$ NMR spectroscopic data $\left(\mathrm{CDCl}_{3}\right)$ for kayeassamin I (1).

\begin{tabular}{|c|c|c|c|c|c|c|}
\hline \multirow[t]{2}{*}{ Position } & \multicolumn{2}{|l|}{$1 a^{a}$} & \multicolumn{2}{|l|}{$1 b^{a}$} & \multicolumn{2}{|l|}{$1^{b}$} \\
\hline & $\delta_{\mathrm{H}}$ & $\delta_{\mathrm{C}}$ & $\delta_{\mathrm{H}}$ & $\delta_{\mathrm{C}}$ & $\delta_{\mathrm{H}}$ & $\delta_{\mathrm{C}}$ \\
\hline 2 & & 159.6 & & 159.6 & & 159.6 \\
\hline 3 & $6.60(\mathrm{br} \mathrm{s})$ & 107.0 & $6.61(\mathrm{~d}, 0.9)$ & 107.2 & $6.61(\mathrm{~s})$ & 107.0 \\
\hline 4 & & 160.6 & & 160.6 & & 160.6 \\
\hline $4 a$ & & 101.0 & & 101.1 & & 101.0 \\
\hline 5 & & 155.9 & & 155.9 & & 155.9 \\
\hline 6 & & 105.8 & & 106.0 & & 105.8 \\
\hline 7 & & 162.9 & & 162.9 & & 162.9 \\
\hline 8 & & 104.5 & & 104.6 & & 104.5 \\
\hline $8 a$ & & 157.2 & & 157.2 & & 157.3 \\
\hline $1^{\prime}$ & 5.43 (br t, ca. 8) & 71.8 & 5.43 (br t, ca. 8) & 71.7 & $5.43(\mathrm{~d}, 8.1)$ & 71.8 \\
\hline $2^{\prime}$ & 1.51, 1.95 (both m) & 30.7 & 1.53, 1.96 (both m) & 30.5 & 1.50, 1.97 (both m) & 30.7 \\
\hline $3^{\prime}$ & $1.11(3 \mathrm{H}, \mathrm{t}, 7.4)$ & 10.2 & $1.09(3 \mathrm{H}, \mathrm{t.} .4 .4)$ & 10.1 & $1.13(3 \mathrm{H}, \mathrm{t}, 7.4)$ & 10.2 \\
\hline $2^{\prime \prime}$ & & 83.0 & & 83.1 & & 83.0 \\
\hline $3^{\prime \prime}$ & $5.53(d, 10.2)$ & 125.0 & $5.55(d, 10.2)$ & 124.8 & $5.54(d, 10.0)$ & 124.9 \\
\hline $4^{\prime \prime}$ & $6.78(d, 10.2)$ & 116.5 & $6.79(d, 10.2)$ & 116.6 & $6.79(d, 10.0)$ & 116.5 \\
\hline $5^{\prime \prime}$ & 1.71, 1.91 (both m) & 41.6 & 1.71, 1.91 (both m) & 41.9 & $1.90(2 \mathrm{H}, \mathrm{m})$ & 41.6 \\
\hline $6^{\prime \prime}$ & $2.09(2 \mathrm{H}, \mathrm{m})$ & 23.0 & $2.09(2 \mathrm{H}, \mathrm{m})$ & 23.2 & $2.09(2 \mathrm{H}, \mathrm{m})$ & 23.0 \\
\hline $7^{\prime \prime}$ & 5.06 (qt, $0.9,7.1$ ) & 123.1 & 5.06 (qt, 0.9, 7.1) & 123.0 & $5.07(t, 7.1)$ & 123.1 \\
\hline $8^{\prime \prime}$ & & 132.6 & & 132.6 & & 132.6 \\
\hline $9^{\prime \prime}$ & $1.64(3 \mathrm{H}, \mathrm{d}, 0.9)$ & 25.6 & $1.67(3 \mathrm{H}, \mathrm{d}, 0.9)$ & 25.6 & $1.64(3 \mathrm{H}, \mathrm{s})$ & 25.6 \\
\hline $10^{\prime \prime}$ & $1.55(3 \mathrm{H}, \mathrm{s})$ & 17.6 & $1.57(3 \mathrm{H}, \mathrm{s})$ & 17.7 & $1.55(3 \mathrm{H}, \mathrm{s})$ & 17.7 \\
\hline $1^{\prime \prime \prime}$ & & 206.4 & & 206.4 & & 206.4 \\
\hline $2^{\prime \prime \prime}$ & $3.26(2 \mathrm{H}, \mathrm{t}, 7.1)$ & 46.7 & $3.26(2 \mathrm{H}, \mathrm{t}, 7.1)$ & 46.7 & $3.27(2 \mathrm{H}, \mathrm{t}, 7.1)$ & 46.7 \\
\hline $3^{\prime \prime \prime}$ & $1.78(2 \mathrm{H}, \mathrm{qt}, 7.4,7.1)$ & 18.0 & $1.78(2 \mathrm{H}, \mathrm{qt}, 7.4,7.1)$ & 18.0 & $1.79(2 \mathrm{H}, \mathrm{m})$ & 18.1 \\
\hline $4^{\prime \prime \prime}$ & $1.04(3 \mathrm{H}, \mathrm{t}, 7.4)$ & 13.8 & $1.03(3 \mathrm{H}, \mathrm{t}, 7.4)$ & 13.8 & $1.05(3 \mathrm{H}, \mathrm{m})$ & 13.8 \\
\hline $2^{\prime \prime}-\mathrm{CH}_{3}$ & $1.52(3 \mathrm{H}, \mathrm{s})$ & 27.2 & $1.48(3 \mathrm{H}, \mathrm{s})$ & 27.5 & $1.51(3 \mathrm{H}, \mathrm{s})$ & 27.3 \\
\hline $7-\mathrm{OH}$ & 14.47 (s) & & $14.47(\mathrm{~s})$ & & 14.48 (brs) & \\
\hline
\end{tabular}

${ }^{a}$ Measured by $800 \mathrm{MHz}$ for ${ }^{1} \mathrm{H}$ NMR and $200 \mathrm{MHz}$ for ${ }^{13} \mathrm{C}$ NMR.

${ }^{b}$ Reported in Win et al. (2008b) by $400 \mathrm{MHz}$ for ${ }^{1} \mathrm{H}$ NMR and $100 \mathrm{MHz}$ for ${ }^{13} \mathrm{C}$ NMR.

of $5 \alpha$-reductases, chronologically named types 1,2 , and $35 \alpha$ reductases, have been described (Yamana et al., 2010; Azzouni et al., 2012; Titus et al., 2014). A type 2 and $35 \alpha$-reductase inhibitor, finasteride, is currently marketed worldwide as a drug for benign prostatic hyperplasia and is also used in the treatment of hair loss (Heinzl, 1999; Tosti and Piraccini, 2000) and in the prevention of prostate cancer (Coltman et al., 1999). Therefore, $5 \alpha$-reductase is considered a useful therapeutic target in the treatment and prevention of the above deceases. In particular, many heterocyclic compounds based on oxygen and nitrogen atoms often have good antiproliferative activity against a variety of solid tumor cell lines and are expected to be seeds of new anticancer agents (Sharma et al., 2018; Petel et al., 2019).

During our characterization studies on bioactive constituents from Thai natural medicines (Manse et al., 2017; Morikawa et al., 2018; Tanabe et al., 2018; Kobayashi et al., 2019), a methanol extract of the flowers of $M$. siamensis was found to inhibit $5 \alpha$ reductase activity $\left(\mathrm{IC}_{50}=2.4 \mu \mathrm{g} / \mathrm{mL}\right)$. In order to investigate new $5 \alpha$-reductase inhibitors, we conducted a search for the bioactive constituents from the flowers of $M$. siamensis.

\section{Isolation}

In our previous report we described the isolation of 26 coumarins: mammeasins A (4, 0.0293\%), B (5, 0.0115\%), C $(6,0.0008 \%)$, and D $(7,0.0047 \%)$, kayeassamins A $(8,0.0578 \%)$, $\mathrm{E}(\mathbf{9}, 0.0113 \%), \mathrm{F}(\mathbf{1 0}, 0.0390 \%)$, and $\mathrm{G}(\mathbf{1 1}, 0.0171 \%)$, surangins B $(12,0.0271 \%), C(13,0.0571 \%)$, and D (14, $0.0632 \%)$, 8-hydroxy-5-methyl-7-(3,7-dimethyl-octa-2,6-dienyl)-9-(2methyl-1-oxobutyl)-4,5-dihydropyrano[4,3,2-de]chromen-2one $(15,0.0015 \%), 8$-hydroxy-5-methyl-7-(3,7-dimethyl-octa2,6-dienyl)-9-(3-methyl-1-oxobutyl)-4,5-dihydropyrano[4,3,2de]chromen-2-one (16, 0.0012\%), mammeas A/AA (17, $0.0494 \%), \mathrm{A} / \mathrm{AB}(\mathbf{1 8}, 0.0048 \%), \mathrm{A} / \mathrm{AC}(\mathbf{1 9}, 0.1056 \%), \mathrm{A} / \mathrm{AD}$ (20, 0.0022\%), E/BA (21, 0.0045\%), E/BB (22, 0.0194\%), A/AA cyclo D $(24,0.0035 \%)$, A/AB cyclo D (25, 0.0097\%), $\mathrm{A} / \mathrm{AC}$ cyclo D (26, 0.0109\%), B/AB cyclo D (27, 0.0016\%), $\mathrm{B} / \mathrm{AC}$ cyclo D $(\mathbf{2 8}, 0.0062 \%), \mathrm{E} / \mathrm{BC}$ cyclo D $(\mathbf{2 9}, 0.0058 \%)$, and deacetylmammea E/BC cyclo D (33, 0.0073\%), as described previously (Morikawa et al., 2012; Ninomiya et al., 2016). In the present study, we additionally isolated kayeassamin I (1, $0.0072 \%)$ and mammeasins $\mathrm{E}(\mathbf{2}, 0.0099 \%)$ and $\mathrm{F}(\mathbf{3}, 0.0015 \%)$, 
TABLE $2 \mid{ }^{1} \mathrm{H}$ and ${ }^{13} \mathrm{C}$ NMR spectroscopic data $\left(\mathrm{CDCl}_{3}\right)$ for mammeasins $\mathrm{E}(\mathbf{2})$ and $\mathrm{F}(\mathbf{3})$.

\begin{tabular}{|c|c|c|c|c|c|c|c|c|}
\hline \multirow[t]{2}{*}{ Position } & \multicolumn{2}{|l|}{$2 a^{a}$} & \multicolumn{2}{|l|}{$2 b^{a}$} & \multicolumn{2}{|l|}{$3 a^{b}$} & \multicolumn{2}{|l|}{$3 b^{b}$} \\
\hline & $\delta_{\mathrm{H}}$ & $\delta_{\mathrm{C}}$ & $\delta_{\mathrm{H}}$ & $\delta_{\mathrm{C}}$ & $\delta_{\mathrm{H}}$ & $\delta_{\mathrm{C}}$ & $\delta_{\mathrm{H}}$ & $\delta_{\mathrm{C}}$ \\
\hline 2 & & 159.6 & & 159.6 & & 159.5 & & 159.5 \\
\hline 3 & $6.61(d, 0.9)$ & 107.0 & $6.59(\mathrm{~d}, 1.0)$ & 107.1 & $6.62(d, 1.0)$ & 107.1 & $6.61(d, 1.0)$ & 107.2 \\
\hline 4 & & 160.7 & & 160.7 & & 160.6 & & 1560.5 \\
\hline $4 a$ & & 101.0 & & 101.1 & & 101.0 & & 101.2 \\
\hline 5 & & 156.0 & & 156.0 & & 155.8 & & 155.8 \\
\hline 6 & & 105.8 & & 106.0 & & 105.9 & & 106.1 \\
\hline 7 & & 163.0 & & 163.0 & & 163.1 & & 163.1 \\
\hline 8 & & 104.5 & & 104.6 & & 104.3 & & 104.3 \\
\hline $8 a$ & & 157.1 & & 157.1 & & 157.0 & & 156.9 \\
\hline $1^{\prime}$ & 5.40 (br t, ca. 8) & 71.8 & 5.40 (br t, ca.8) & 71.7 & $5.43(\mathrm{~m})$ & 71.8 & $5.43(\mathrm{~m})$ & 71.8 \\
\hline $2^{\prime}$ & $1.53,1.96$ (both m) & 30.7 & 1.53, 1.96 (both m) & 30.6 & 1.52, 1.96 (both m) & 30.7 & 1.52, 1.96 (both m) & 30.6 \\
\hline $3^{\prime}$ & $1.12(3 \mathrm{H}, \mathrm{t}, 7.3)$ & 10.2 & $1.09(3 \mathrm{H}, \mathrm{t}, 7.1)$ & 10.1 & $1.12(3 \mathrm{H}, \mathrm{t}, 7.1)$ & 10.2 & $1.10(3 \mathrm{H}, \mathrm{t}, 7.1)$ & 10.1 \\
\hline $2^{\prime \prime}$ & & 83.0 & & 83.1 & & 83.0 & & 83.1 \\
\hline $3^{\prime \prime}$ & $5.53(d, 10.2)$ & 125.0 & $5.54(d, 10.2)$ & 124.9 & $5.54(d, 10.2)$ & 124.9 & $5.54(d, 10.2)$ & 124.8 \\
\hline $4^{\prime \prime}$ & $6.79(d, 10.2)$ & 116.5 & $6.78(d, 10.2)$ & 116.5 & $6.79(d, 10.2)$ & 116.6 & $6.79(d, 10.2)$ & 116.6 \\
\hline $5^{\prime \prime}$ & $1.71,1.90$ (both m) & 41.6 & $1.71,1.90$ (both m) & 41.8 & $1.71,1.91$ (both m) & 41.7 & 1.71, 1.91 (both m) & 41.9 \\
\hline $6^{\prime \prime}$ & $2.08(2 \mathrm{H}, \mathrm{m})$ & 23.0 & $2.08(2 \mathrm{H}, \mathrm{m})$ & 23.2 & $2.09(2 \mathrm{H}, \mathrm{m})$ & 23.0 & $2.09(2 \mathrm{H}, \mathrm{m})$ & 23.3 \\
\hline $7^{\prime \prime}$ & 5.06 (qt, 1.0, 7.1) & 123.1 & $5.06(q t, 1.0,7.1)$ & 123.0 & $5.06(\mathrm{dt}, 1.3,7.1)$ & 123.1 & $5.06(q t, 1.3,7.1)$ & 123.0 \\
\hline $8^{\prime \prime}$ & & 132.6 & & 132.5 & & 132.6 & & 132.6 \\
\hline $9^{\prime \prime}$ & $1.64(3 \mathrm{H}, \mathrm{d}, 1.0)$ & 25.5 & $1.67(3 \mathrm{H}, \mathrm{d}, 1.0)$ & 25.6 & $1.64(3 \mathrm{H}, \mathrm{br} \mathrm{s})$ & 25.6 & $1.67(3 \mathrm{H}, \mathrm{br} \mathrm{s})$ & 25.6 \\
\hline $10^{\prime \prime}$ & $1.52(3 \mathrm{H}, \mathrm{s})$ & 17.6 & $1.54(3 \mathrm{H}, \mathrm{s})$ & 17.7 & $1.57(3 \mathrm{H}, \mathrm{s})$ & 17.6 & $1.57(3 \mathrm{H}, \mathrm{s})$ & 17.7 \\
\hline $1^{\prime \prime \prime}$ & & 206.2 & & 206.2 & & 210.7 & & 210.7 \\
\hline $2^{\prime \prime \prime}$ & $3.14(2 \mathrm{H}, \mathrm{d}, 6.7)$ & 53.6 & $3.14(2 \mathrm{H}, \mathrm{d}, 6.7)$ & 53.6 & $3.89(\mathrm{~m})$ & 47.0 & $3.89(\mathrm{~m})$ & 47.0 \\
\hline $3^{\prime \prime \prime}$ & $2.27(\mathrm{~m})$ & 25.6 & $2.27(\mathrm{~m})$ & 25.5 & $1.25(3 \mathrm{H}, \mathrm{d}, 6.7)$ & 16.6 & $1.26(3 \mathrm{H}, \mathrm{d}, 6.7)$ & 16.6 \\
\hline $4^{\prime \prime \prime}$ & $1.03(3 \mathrm{H}, \mathrm{d}, 6.6)$ & 22.6 & $1.03(3 \mathrm{H}, \mathrm{d}, 6.6)$ & 22.6 & 1.46, 1.89 (both m) & 27.2 & $1.46,1.89$ (each m) & 27.2 \\
\hline $5^{\prime \prime \prime}$ & $1.03(3 \mathrm{H}, \mathrm{d}, 6.6)$ & 22.6 & $1.03(3 \mathrm{H}, \mathrm{d}, 6.6)$ & 22.6 & $0.98(3 \mathrm{H}, \mathrm{t}, 7.5)$ & 11.7 & $0.98(3 \mathrm{H}, \mathrm{t}, 7.5)$ & 11.7 \\
\hline $2^{\prime \prime \prime}-\mathrm{CH}_{3}$ & $1.51(3 \mathrm{H}, \mathrm{s})$ & 27.3 & $1.48(3 \mathrm{H}, \mathrm{s})$ & 27.5 & $1.52(3 \mathrm{H}, \mathrm{br} \mathrm{s})$ & 27.3 & $1.47(3 \mathrm{H}, \mathrm{br} \mathrm{s})$ & 27.5 \\
\hline $7-\mathrm{OH}$ & $14.51(\mathrm{~s})$ & & $14.51(\mathrm{~s})$ & & $14.44(\mathrm{~s})$ & & $14.44(\mathrm{~s})$ & \\
\hline
\end{tabular}

a Measured by $700 \mathrm{MHz}$ for ${ }^{1} \mathrm{H}$ NMR and $175 \mathrm{MHz}$ for ${ }^{13} \mathrm{C}$ NMR.

${ }^{b}$ Measured by $800 \mathrm{MHz}$ for ${ }^{1} \mathrm{H}$ NMR and $200 \mathrm{MHz}$ for ${ }^{13} \mathrm{C}$ NMR.

from the methanol extract of $M$. siamensis flowers as shown in Figure 1, together with six coumarins: mammeas E/BC (23, $0.0076 \%)$ and $\mathrm{E} / \mathrm{BD}$ cyclo $\mathrm{D}(\mathbf{3 0}, 0.0015 \%)$, deacetylmammeas E/AA cyclo D (31, 0.0005\%) and E/BB cyclo D (32, 0.0023\%), and mammeas A/AA cyclo F (34, 0.0010\%) and A/AC cyclo F $(35,0.0068 \%)$, using normal-phase silica gel and reversed-phase ODS column chromatographic purification steps, and finally by HPLC (Figure 2).

\section{Structures of Kayeassamin I (1) and Mammeasins E (2) and F (3)}

Compound $\mathbf{1}$ was obtained as pale yellow oil with a negative optical rotation $\left([\alpha]_{\mathrm{D}}^{25}-50.4\right.$ in $\left.\mathrm{CHCl}_{3}\right)$, and its molecular formula was deduced to be $\mathrm{C}_{26} \mathrm{H}_{32} \mathrm{O}_{6}$ by high-resolution ESIMS (HRESIMS) measurement. As shown in Figure 3, the HPLC analysis suggested that $\mathbf{1}$ was obtained as an inseparable mixture (ca. 1:1 ratio). The ${ }^{1} \mathrm{H}$ and ${ }^{13} \mathrm{C}$ NMR spectra spectroscopic properties (Table $\mathbf{1}, \mathrm{CDCl}_{3}$ ) of $\mathbf{1}$, which were assigned with the aid of DEPT, DQF-COSY, HSQC, and HMBC experiments, were in accordance with those of kayeassamin I except for the observation of duplicate signals (1a and 1b) measured by high resolution $800 \mathrm{MHz}$ NMR spectrometer: two primary, a tertiary, and two vinyl methyls [1a: $\delta 1.04\left(3 \mathrm{H}, \mathrm{t}, J=7.4 \mathrm{~Hz}, \mathrm{H}_{3}-4^{\prime \prime \prime}\right)$, $1.11\left(3 \mathrm{H}, \mathrm{t}, J=7.4 \mathrm{~Hz}, \mathrm{H}_{3}-3^{\prime}\right), 1.52\left(3 \mathrm{H}, \mathrm{s}, 2^{\prime \prime}-\mathrm{CH}_{3}\right), 1.55(3 \mathrm{H}$, s, $\left.\mathrm{H}_{3}-10^{\prime \prime}\right), 1.64\left(3 \mathrm{H}, \mathrm{d}, J=0.9 \mathrm{~Hz}, \mathrm{H}_{3}-9^{\prime \prime}\right) ; \mathbf{l b}: \delta 1.03(3 \mathrm{H}, \mathrm{t}$, $\left.J=7.4 \mathrm{~Hz}, \mathrm{H}_{3}-4^{\prime \prime \prime}\right), 1.09\left(3 \mathrm{H}, \mathrm{t}, J=7.4 \mathrm{~Hz}, \mathrm{H}_{3}-3^{\prime}\right), 1.48(3 \mathrm{H}$, s, $\left.2^{\prime \prime}-\mathrm{CH}_{3}\right), 1.57\left(3 \mathrm{H}, \mathrm{s}, \mathrm{H}_{3}-10^{\prime \prime}\right), 1.67\left(3 \mathrm{H}, \mathrm{d}, J=0.9 \mathrm{~Hz}, \mathrm{H}_{3}-\right.$ $\left.9^{\prime \prime}\right)$ ], five methylenes [1a: $\delta 1.51,1.95\left(1 \mathrm{H}\right.$ each, both $\mathrm{m}, \mathrm{H}_{2}$ $\left.2^{\prime}\right), 1.71,1.91$ ( $1 \mathrm{H}$ each, both $\left.\mathrm{m}, \mathrm{H}_{2}-5^{\prime \prime}\right), 1.78(2 \mathrm{H}, \mathrm{qt}, J=7.4$, $\left.7.1 \mathrm{~Hz}, \mathrm{H}_{2}-3^{\prime \prime \prime}\right), 2.09\left(2 \mathrm{H}, \mathrm{m}, \mathrm{H}_{2}-6^{\prime \prime}\right), 3.26(2 \mathrm{H}, \mathrm{t}, J=7.1 \mathrm{~Hz}$, $\left.\mathrm{H}_{2}-2^{\prime \prime \prime}\right)$; $1 \mathbf{b}: \delta 1.53,1.96$ ( $1 \mathrm{H}$ each, both $\left.\mathrm{m}, \mathrm{H}_{2}-2^{\prime}\right), 1.71,1.91$ ( $1 \mathrm{H}$ each, both $\left.\mathrm{m}, \mathrm{H}_{2}-5^{\prime \prime}\right), 1.78\left(2 \mathrm{H}, \mathrm{qt}, J=7.4,7.1 \mathrm{~Hz}, \mathrm{H}_{2}\right.$ $\left.\left.3^{\prime \prime \prime}\right), 2.09\left(2 \mathrm{H}, \mathrm{m}, \mathrm{H}_{2}-6^{\prime \prime}\right), 3.26\left(2 \mathrm{H}, \mathrm{t}, J=7.1 \mathrm{~Hz}, \mathrm{H}_{2}-2^{\prime \prime \prime}\right)\right]$, a methine bearing an oxygen function [1a: $\delta 5.43(1 \mathrm{H}$, br t, $J=$ ca. $\left.8 \mathrm{~Hz}, \mathrm{H}_{-1}^{\prime}\right)$; $\mathbf{1 b}: \delta 5.43\left(1 \mathrm{H}\right.$, br t, $\left.\left.J=c a .8 \mathrm{~Hz}, \mathrm{H}-1^{\prime}\right)\right]$, four olefinic protons [1a: $\delta 5.06\left(1 \mathrm{H}, \mathrm{qt}, J=0.9,7.1 \mathrm{~Hz}, \mathrm{H}-7^{\prime \prime}\right), 5.53$ $\left(1 \mathrm{H}, \mathrm{d}, J=10.2 \mathrm{~Hz}, \mathrm{H}-3^{\prime \prime}\right), 6.60(1 \mathrm{H}$, br s, H-3), $6.78(1 \mathrm{H}, \mathrm{d}$, $\left.J=10.2 \mathrm{~Hz}, \mathrm{H}-4^{\prime \prime}\right)$; $\mathbf{1 b}: \delta 5.06\left(1 \mathrm{H}, \mathrm{qt}, J=0.9,7.1 \mathrm{~Hz}, \mathrm{H}-7^{\prime \prime}\right)$, 
<smiles>CCCC(=O)c1c(O)c2c(c3oc(=O)cc(C(O)CC)c13)OC(C)([CH+]CC=C([O-])[O-])C=C2</smiles>

kayeassamin I (1)

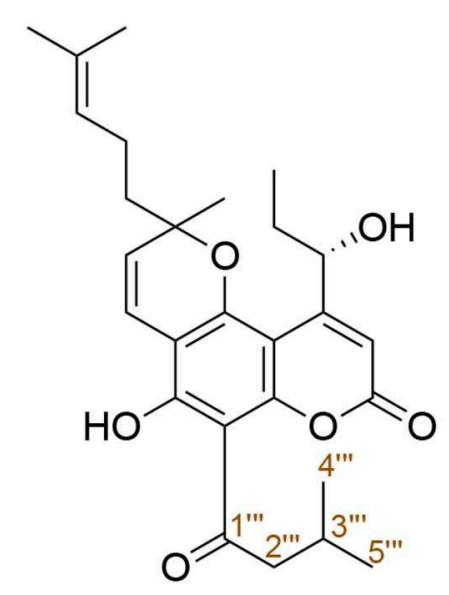

mammeasin $E(2)$

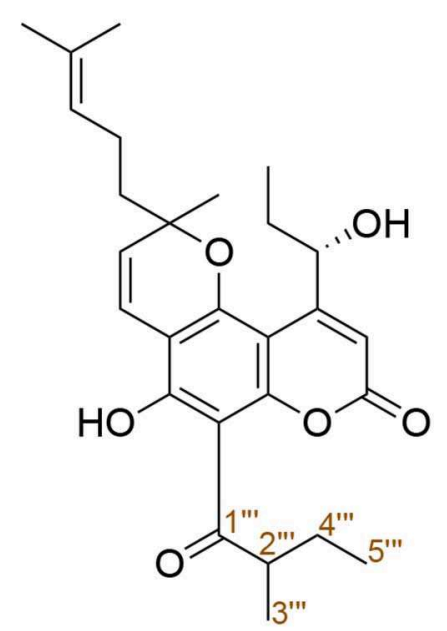

mammeasin F (3)

FIGURE 1 | Structures of kayeassamin I (1) and mammeasins E (2) and F (3).

$5.55\left(1 \mathrm{H}, \mathrm{d}, J=10.2 \mathrm{~Hz}, \mathrm{H}-3^{\prime \prime}\right), 6.61(1 \mathrm{H}, \mathrm{d}, J=0.9 \mathrm{~Hz}, \mathrm{H}-$ 3), $\left.6.79\left(1 \mathrm{H}, \mathrm{d}, J=10.2 \mathrm{~Hz}, \mathrm{H}-4^{\prime \prime}\right)\right]$, and a hydrogen-bonded hydroxy proton [1a: $\delta 14.47(1 \mathrm{H}, \mathrm{s}, 7-\mathrm{OH})$; $1 \mathbf{b}: \delta 14.47(1 \mathrm{H}$, s, 7-OH)]. This evidence allowed us to revise the structure of kayeassamin I as a mixture (1a and $\mathbf{1 b}$ ) of ca. 1:1 inseparable stereoisomers in the $2^{\prime \prime}$ position. The absolute configuration of the $1^{\prime}$-position in $\mathbf{1}$ has been assumed to be $S$ by comparison of the optical rotation with that of similar compounds (Win et al., 2008b). To confirm the stereochemistry, we carried out chemical correlation between $\mathbf{1}$ and kayeassamin A (8), which has been reported to be in the $1^{\prime} S$ form by the modified Mosher's method (Win et al., 2008a). Thus, oxidation of $\mathbf{8}$ with 2,3-dichloro5,6-dicyano-p-benzoquinone (DDQ) gave $\mathbf{1}$. Consequently, the absolute configuration in the $1^{\prime}$ position of $\mathbf{1}$ was confirmed to be $S$.

Mammeasin E (2) was also obtained as an inseparable mixture (ca. 1:1 ratio, Figure $\mathbf{S 1}$ ) with a negative optical rotation $\left([\alpha]_{\mathrm{D}}^{25}-58.9\right.$ in $\left.\mathrm{CHCl}_{3}\right)$. In the negative-ion ESIMS of 2, a quasimolecular ion peak was observed at $\mathrm{m} / z 453[\mathrm{M}-\mathrm{H}]^{-}$, and HRESIMS analysis indicated the molecular formula was $\mathrm{C}_{27} \mathrm{H}_{34} \mathrm{O}_{6}$. The ${ }^{1} \mathrm{H}$ and ${ }^{13} \mathrm{C}$ NMR spectra (Table 2, $\mathrm{CDCl}_{3}$ ) of 2 were similar to those of $\mathbf{1}$, except for the signals due to the 3-methyl-1-oxobutyl moiety in the 8-position [2a: $\delta 1.03(6 \mathrm{H}$, $\mathrm{d}, J=6.6 \mathrm{~Hz}, \mathrm{H}_{3}-4^{\prime \prime \prime}$ and $\left.\mathrm{H}_{3}-5^{\prime \prime \prime}\right), 2.27\left(1 \mathrm{H}, \mathrm{m}, \mathrm{H}-3^{\prime \prime \prime}\right), 3.14$ $\left(2 \mathrm{H}, \mathrm{d}, J=6.7 \mathrm{~Hz}, \mathrm{H}_{2}-2^{\prime \prime \prime}\right) ; 2 \mathrm{~b}: \delta 1.03\left(6 \mathrm{H}, \mathrm{d}, J=6.6 \mathrm{~Hz}, \mathrm{H}_{3}-\right.$ $4^{\prime \prime \prime}$ and $\left.\mathrm{H}_{3}-5^{\prime \prime \prime}\right), 2.27\left(1 \mathrm{H}, \mathrm{m}, \mathrm{H}-3^{\prime \prime \prime}\right), 3.14(2 \mathrm{H}, \mathrm{d}, J=6.7 \mathrm{~Hz}$, $\left.\mathrm{H}_{2}-2^{\prime \prime \prime}\right)$ ] instead of the 1-oxobutyl moiety of $\mathbf{1}$. As shown in Figure S2, the connectivity of the quaternary carbons in $\mathbf{2}$ were elucidated on the basis of DQF-COSY and HMBC experiments. Thus, the DQF-COSY experiment on 2 indicated the presence of the following partial structures: C-1'-C-3'; C- $3^{\prime \prime}-\mathrm{C}-4^{\prime \prime}$; C$5^{\prime \prime}-\mathrm{C}-7^{\prime \prime}$; and $\mathrm{C}-2^{\prime \prime \prime}-\mathrm{C}-5^{\prime \prime \prime}$ shown in bold lines. In the HMBC experiment, long-range correlations were observed between the following proton and carbon pairs: $\mathrm{H}-3$ [2a: $\delta 6.61(1 \mathrm{H}, \mathrm{d}, J=$ $0.9 \mathrm{~Hz}) ; 2 \mathbf{b}: \delta 6.59(1 \mathrm{H}, \mathrm{d}, J=1.0 \mathrm{~Hz})]$ and C-2 $\left(2 \mathrm{a}: \delta_{\mathrm{C}} 159.6\right.$; 2b: $\delta_{\mathrm{C}}$ 159.6), C-4a $\left(2 \mathbf{a}: \delta_{\mathrm{C}} 101.0 ; \mathbf{2 b}: \delta_{\mathrm{C}} 101.1\right)$; the hydrogenbonded hydroxy proton [2a: $\delta 14.51(1 \mathrm{H}, \mathrm{s}) ; \mathbf{2 b}: \delta 14.51(1 \mathrm{H}, \mathrm{s})]$ and C-6 (2a: $\left.\delta_{\mathrm{C}} 105.8 ; 2 \mathbf{b}: \delta_{\mathrm{C}} 106.0\right), \mathrm{C}-7\left(\mathbf{2 a}: \delta_{\mathrm{C}} 163.0 ; \mathbf{2 b}: \delta_{\mathrm{C}}\right.$ 163.0), C-8 (2a: $\delta_{\mathrm{C}} 104.5$; 2b: $\left.\delta_{\mathrm{C}} 104.6\right)$; H-1' [2a: $\delta 5.40(1 \mathrm{H}$, br t, $J=c a .8 \mathrm{~Hz}) ; 2 \mathrm{~b}: \delta 5.40(1 \mathrm{H}$, br t, $J=c a .8 \mathrm{~Hz})]$ and C$3\left(2 \mathbf{a}: \delta_{\mathrm{C}} 107.0 ; 2 \mathbf{b}: \delta_{\mathrm{C}} 107.1\right), \mathrm{C}-4 \mathrm{a} ; \mathrm{H}-3^{\prime \prime \prime}$ [2a: $\delta 5.53(1 \mathrm{H}, \mathrm{d}, J$ $=10.2 \mathrm{~Hz}) ; 2 \mathbf{b}: \delta 5.54(1 \mathrm{H}, \mathrm{d}, J=10.2 \mathrm{~Hz})]$ and C-6, C-2" $(2 \mathrm{a}:$ $\left.\delta_{\mathrm{C}} 83.0 ; 2 \mathbf{b}: \delta_{\mathrm{C}} 83.1\right), 2^{\prime \prime}-\mathrm{CH}_{3}\left(2 \mathbf{a}: \delta_{\mathrm{C}} 27.3 ; \mathbf{2 b}: \delta_{\mathrm{C}} 27.5\right) ; \mathrm{H}-4^{\prime \prime}$ [2a: $\delta 6.79(1 \mathrm{H}, \mathrm{d}, J=10.2 \mathrm{~Hz}) ; 2 \mathbf{b}: \delta 6.78(1 \mathrm{H}, \mathrm{d}, J=10.2 \mathrm{~Hz})]$ and C-5 (2a: $\left.\delta_{\mathrm{C}} 156.0 ; 2 \mathbf{b}: \delta_{\mathrm{C}} 156.0\right), \mathrm{C}-6 ; \mathrm{H}_{2}-5^{\prime \prime}$ [2a: $\delta 1.71,1.90$ (1H each, both $\mathrm{m}) ; 2 \mathbf{b}: \delta 1.71,1.90(1 \mathrm{H}$ each, both $\mathrm{m})]$ and $\mathrm{C}-$ $2^{\prime \prime}, 2^{\prime \prime}-\mathrm{CH}_{3} ; \mathrm{H}-7^{\prime \prime}[\mathbf{2 a}: \delta 5.06(1 \mathrm{H}, \mathrm{qt}, J=1.0,7.1 \mathrm{~Hz}) ; 2 \mathbf{b}: \delta 5.06$ $(1 \mathrm{H}, \mathrm{qt}, J=1.0,7.1 \mathrm{~Hz})]$ and $\mathrm{C}-9^{\prime \prime}\left(2 \mathbf{a}: \delta_{\mathrm{C}} 25.5 ; \mathbf{2 b}: \delta_{\mathrm{C}} 25.6\right)$, C-10" $\left(2 \mathbf{a}: \delta_{\mathrm{C}} 17.6 ; 2 \mathbf{b}: \delta_{\mathrm{C}} 17.7\right)$; H-9" [2a: $\delta 1.64(3 \mathrm{H}, \mathrm{d}, J=$ $1.0 \mathrm{~Hz}) ; 2 \mathrm{~b}: \delta 1.67(3 \mathrm{H}, \mathrm{d}, J=1.0 \mathrm{~Hz})]$ and C-7" $\left(2 \mathrm{a}: \delta_{\mathrm{C}} 123.1\right.$; 2b: $\delta_{\mathrm{C}}$ 123.0), C-8" $\left(2 \mathrm{a}: \delta_{\mathrm{C}} 132.6 ; 2 \mathbf{b}: \delta_{\mathrm{C}} 132.5\right), \mathrm{C}-10^{\prime \prime} ; \mathrm{H}-10^{\prime \prime}$ [2a: $\delta 1.52(3 \mathrm{H}, \mathrm{s}) ; 2 \mathbf{b}: \delta 1.54(3 \mathrm{H}, \mathrm{s})]$ and $\mathrm{C}-7^{\prime \prime}-9^{\prime \prime}$; and $\mathrm{H}_{2}-$ $2^{\prime \prime \prime}$ and $\mathrm{C}-1^{\prime \prime \prime}\left(2 \mathbf{a}: \delta_{\mathrm{C}} 206.2 ; \mathbf{2 b}: \delta_{\mathrm{C}} 206.2\right)$. On the other hand, the molecular formula of mammeasin $\mathrm{F}$ (3) was determined to be the same as that of $2, \mathrm{C}_{27} \mathrm{H}_{34} \mathrm{O}_{6}$, by HRESIMS measurement. The ${ }^{1} \mathrm{H}$ and ${ }^{13} \mathrm{C}$ NMR spectroscopic properties (Table 2, $\mathrm{CDCl}_{3}$ ) of 3 , which were observed to be duplicate signals caused by its inseparable mixture (ca. 1: 1 ratio, Figure S1), were quite similar to those of 2 except for the signals due to the 2-methyl-1-oxobutyl moiety in the 8-position [3a: $\delta 0.98\left(3 \mathrm{H}, \mathrm{t}, J=7.5 \mathrm{~Hz}, \mathrm{H}_{3}-5^{\prime \prime \prime}\right)$, $1.25\left(3 \mathrm{H}, \mathrm{d}, J=6.7 \mathrm{~Hz}, \mathrm{H}_{3}-3^{\prime \prime \prime}\right), 1.46,1.89$ (each $1 \mathrm{H}$, both $\mathrm{m}$, $\left.\mathrm{H}_{2}-4^{\prime \prime \prime}\right), 3.89$ ( $\left.1 \mathrm{H}, \mathrm{m}, \mathrm{H}_{2}-2^{\prime \prime \prime}\right) ; 3 \mathbf{b}: \delta 0.98\left(3 \mathrm{H}, \mathrm{t}, J=7.5 \mathrm{~Hz}, \mathrm{H}_{3}-\right.$ $\left.5^{\prime \prime \prime}\right), 1.26\left(3 \mathrm{H}, \mathrm{d}, J=6.7 \mathrm{~Hz}, \mathrm{H}_{3}-3^{\prime \prime \prime}\right), 1.46,1.89$ (each $1 \mathrm{H}$, both $\mathrm{m}$, $\left.\mathrm{H}_{2}-4^{\prime \prime \prime}\right), 3.89\left(1 \mathrm{H}, \mathrm{m}, \mathrm{H}_{2}-2^{\prime \prime \prime}\right)$ ]. Finally, 2 and 3 were derived by DDQ oxidation of surangins D (14) (Ngo et al., 2010) and C (13) (Verotta et al., 2004; Yagi et al., 2006), respectively. Based on this 


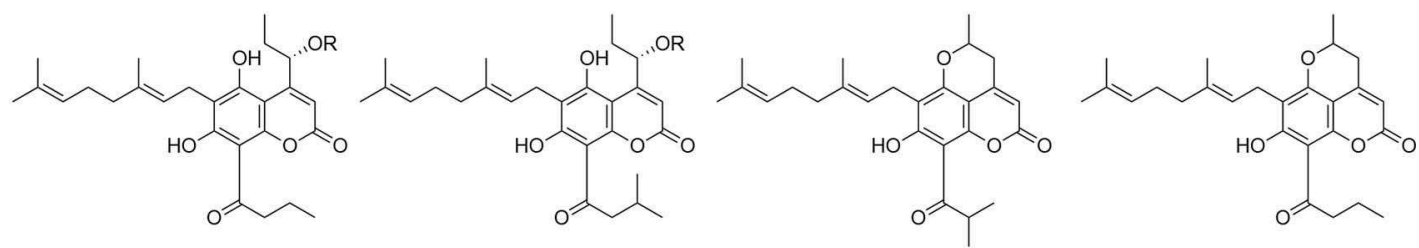

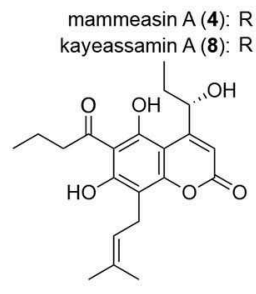

kayeassamin $\mathrm{E}(9)$

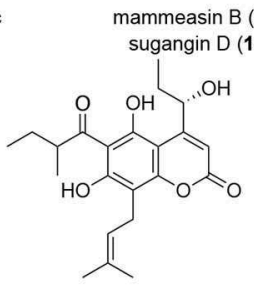

kayeassamin $F(10)$<smiles>CCC(C)C(=O)c1c(O)c(CC=C(C)CCC=C(C)C)c2c3c(cc(=O)oc13)CC(C)O2</smiles>

mammeasin C (6)

mammeasin $D(7)$<smiles>[R]OC(CC)c1cc(=O)oc2c(C(=O)C(C)CC)c(O)c(CC=C(C)CCC=C(C)C)c(O)c12</smiles>

kayeassamin $\mathrm{G}$ (11)

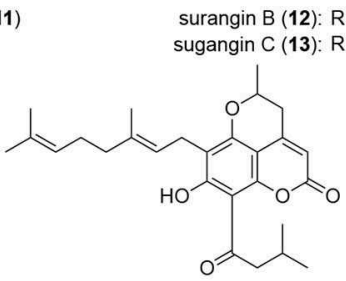

8-hydroxy-5-methyl-7-(3,7-dimethylocta-2,6-dienyl)-9-(2-methyl-1oxobutyl)-4,5-dihydropyranol[4,3,2-de]chromen-2-one (15)

8-hydroxy-5-methyl-7-(3,7-dimethylocta-2,6-dienyl)-9-(3-methyl-1oxobutyl)-4,5-dihydropyranol[4,3,2-de]chromen-2-one (16)<smiles></smiles>

\footnotetext{
mammea AVAA (17)

mammea A/AB (18)

mammea A/AC (19)
}

mammea A/AD (20)

mammea E/BA (21)<smiles>CCC(C)C(=O)c1c(O)c(C(=O)CCC=C(C)C)c(O)c2c(C(CC)OC(C)=O)cc(=O)oc12</smiles><smiles>CCCC(=O)c1c(O)c(C(=O)CCC=C(C)C)c(O)c2c(C(CC)OC(C)=O)cc(=O)oc12</smiles><smiles>CCC(C)C(=O)c1c2c(c3oc(=O)cc(-c4ccccc4)c3c1O)C=CC(C)(C)O2</smiles><smiles></smiles><smiles>CCCc1cc(=O)oc2c3c(c(C(=O)C(C)CC)c(O)c12)OC(C)(C)C=C3</smiles><smiles>CCCC(=O)c1c2c(c3oc(=O)cc(CCC)c3c1O)C=CC(C)(C)O2</smiles><smiles>CCCC(=O)c1c(O)c2c(c3c(C(CC)OC)cc(=O)oc13)OC(C)(C)C=C2</smiles><smiles>CCC(OC(C)=O)c1cc(=O)oc2c(C(=O)C(C)C)c(O)c3c(c12)OC(C)(C)C=C3</smiles><smiles>CC[C@H](O)c1cc(=O)oc2c3c(c(C(=O)CC(C)C)c(O)c12)OC(C)(C)C=C3</smiles>

mammea B/AB cyclo D (27) mammea B/AC cyclo D (28) mammea E/BC cyclo D (29) mammea E/BD cyclo D (30) deacetylmammea E/AA cyclo D (31)<smiles>CCC(C)C(=O)c1c(O)c2c(c3c([C@H](O)CC)cc(=O)oc13)OC(C)(C)C=C2</smiles><smiles>CCCC(=O)c1c(O)c2c(c3c([C@H](O)CC)cc(=O)oc13)OC(C)(C)C=C2</smiles><smiles></smiles>

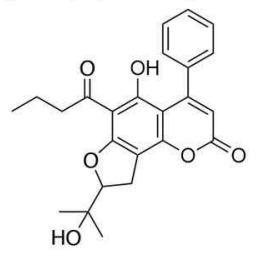

deacetylmammea E/BB cyclo $D(\mathbf{3 2})$ deacetylmammea $E / B C$ cyclo $D(\mathbf{3 3})$ mammea A/AA cyclo $F(\mathbf{3 4})$ mammea $A / A C$ cyclo $F(35)$

FIGURE 2 | Coumarin constituents (4-35) from the flowers of $M$. siamensis. 


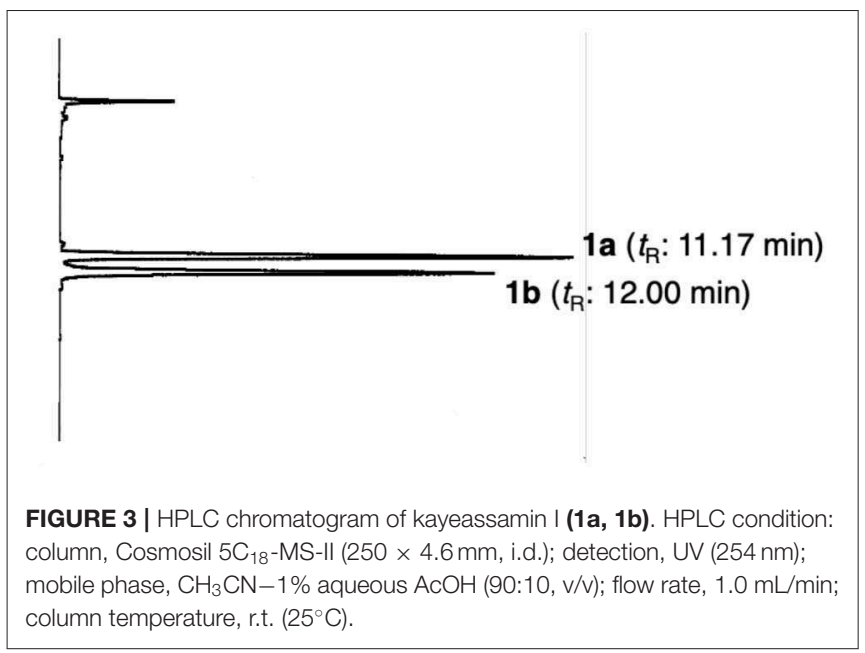

evidence, the stereostructures of $\mathbf{2}$ and $\mathbf{3}$ were determined to be as shown.

\section{Effects of Coumarin Constituents of the Flowers of $M$. siamensis on Testosterone $5 \alpha$-Reductase}

To characterize the active constituents of this plant material, the inhibitory effects of 30 isolates $(1-13,17-20,22-29,31-$ 35) against $5 \alpha$-reductase were examined. As shown in Table 3, mammeasins $\mathrm{E}(2,22.6 \mu \mathrm{M}), \mathrm{A}(4,19.0 \mu \mathrm{M})$, and $\mathrm{B}(5,24.0 \mu \mathrm{M})$, kayeassamins $\mathrm{E}(\mathbf{9}, 33.8 \mu \mathrm{M}), \mathrm{F}(\mathbf{1 0}, 15.9 \mu \mathrm{M})$, and $\mathrm{G}(\mathbf{1 1}$, $17.7 \mu \mathrm{M})$, surangin $\mathrm{C}(\mathbf{1 3}, 5.9 \mu \mathrm{M})$, and mammeas A/AA (17, $19.5 \mu \mathrm{M}), \mathrm{E} / \mathrm{BB}(22,16.8 \mu \mathrm{M})$, and A/AA cyclo F $(34,23.6 \mu \mathrm{M})$, were found to inhibit testosterone $5 \alpha$-reductase (Table S1).

\section{CONCLUSIONS}

The structures of geranylated coumarin constituents, kayeassamin I (1) and mammeasins E (2) and F (3), newly isolated from the methanol extract of the flowers of $M$. siamensis, were determined. Of the isolated coumarins, mammeasins E $(2,22.6 \mu \mathrm{M}), \mathrm{A}(4,19.0 \mu \mathrm{M})$, and B (5, $24.0 \mu \mathrm{M})$, kayeassamins $\mathrm{E}(\mathbf{9}, 33.8 \mu \mathrm{M}), \mathrm{F}(\mathbf{1 0}, 15.9 \mu \mathrm{M})$, and $\mathrm{G}(11,17.7 \mu \mathrm{M})$, surangin $\mathrm{C}(13,5.9 \mu \mathrm{M})$, and mammeas A/AA $(17,19.5 \mu \mathrm{M}), \mathrm{E} / \mathrm{BB}(22,16.8 \mu \mathrm{M})$, and A/AA cyclo F $(34,23.6 \mu \mathrm{M})$ were active $5 \alpha$-reductase inhibitors. Although the intensity of the $5 \alpha$-reductase inhibitory activity of these coumarins is moderate compared to a positive control having a steroid skeleton finasteride, to the best of our knowledge, there are few reports of the $5 \alpha$-reductase inhibitors with non-steroidal skeletons (Dörsam and Altwein, 2009; Aggarwal et al., 2010; Chaudhary and Turner, 2010; Wu and Kapoor, 2013). Therefore, these active coumarins may be useful candidates for seed compounds of new non-steroidal $5 \alpha$ reductase inhibitors. Further studies are required to elucidate the detailed structure activity relationships as well as the
TABLE 3 | IC $\mathrm{C}_{50}$ values of coumarin constituents from $M$. siamensis on testosterone $5 \alpha$-reductase.

\begin{tabular}{|c|c|}
\hline & $I C_{50}(\mu M)$ \\
\hline Kayeassamin I (1) & $>100(37.5)^{\mathrm{a}}$ \\
\hline Mammeasin E (2) & 22.6 \\
\hline Mammeasin F (3) & $>100(14.9)^{\mathrm{a}}$ \\
\hline Mammeasin A (4) & 19.0 \\
\hline Mammeasin B (5) & 24.0 \\
\hline Mammeasin C (6) & 91.9 \\
\hline Mammeasin D (7) & $>100(16.4)^{a}$ \\
\hline Kayeassamin A (8) & $>100(20.2)^{\mathrm{a}}$ \\
\hline Kayeassamin E (9) & 33.8 \\
\hline Kayeassamin F (10) & 15.9 \\
\hline Kayeassamin G (11) & 17.7 \\
\hline Surangin B (12) & $>100(38.5)^{a}$ \\
\hline Surangin C (13) & 5.9 \\
\hline Mammea A/AA (17) & 19.5 \\
\hline Mammea A/AB (18) & $>100(23.3)^{a}$ \\
\hline Mammea A/AC (19) & $>100(41.5)^{\mathrm{a}}$ \\
\hline Mammea A/AD (20) & $>100(30.3)^{\mathrm{a}}$ \\
\hline Mammea E/BB (22) & 16.8 \\
\hline Mammea E/BC (23) & $>100(19.1)^{a}$ \\
\hline Mammea A/AA cyclo D (24) & $>100(38.3)^{a}$ \\
\hline Mammea A/AB cyclo D (25) & $>100(6.7)$ \\
\hline Mammea A/AC cyclo D (26) & $>100(32.0)^{\mathrm{a}}$ \\
\hline Mammea B/AB cyclo D (27) & $>100(40.7)^{a}$ \\
\hline Mammea B/AC cyclo D (28) & $>100(27.3)^{\mathrm{a}}$ \\
\hline Mammea E/BC cyclo D (29) & $>100(31.9)^{a}$ \\
\hline Deacetylmammea E/AA cyclo D (31) & $>100(37.1)^{a}$ \\
\hline Deacetylmammea E/BB cyclo D (32) & $>100(31.9)^{\mathrm{a}}$ \\
\hline Deacetylmammea E/BC cyclo D (33) & $>100(40.8)^{\mathrm{a}}$ \\
\hline Mammea A/AA cyclo F (34) & 23.6 \\
\hline Mammea AVAC cyclo F (35) & 83.8 \\
\hline Finasteride ${ }^{b}$ & 0.12 \\
\hline
\end{tabular}

Each value represents the mean \pm S.E.M. $(N=3-4)$.

${ }^{a}$ Values in parentheses present of control of cell viability at $100 \mu \mathrm{M}$.

${ }^{b}$ Commercial finasteride was purchased from Sigma-Aldrich Co. LLC (St. Louis, USA).

mode of action including the enzymatic inhibitory activity of these coumarins.

\section{DATA AVAILABILITY STATEMENT}

All datasets generated for this study are included in the article/Supplementary Material.

\section{AUTHOR CONTRIBUTIONS}

TM, FL, YM, HS, SS, and KN performed the experiments. TM, $\mathrm{OM}$, and $\mathrm{KN}$ conceived and designed the experiments. SC and YP collected and identified the plant material. TM and FL wrote the paper. All authors have approved the final version of the manuscript. 


\section{FUNDING}

This work was supported in part by the JSPS KAKENHI, Japan [Grant Numbers 18K06726 (TM) and 18K06739 (KN)].

\section{ACKNOWLEDGMENTS}

The authors gratefully thank the Division of Joint Research Center, Kindai University for the NMR and MS measurements. We would like to thank Editage (www.editage.com) for English language editing.

\section{REFERENCES}

Aggarwal, S., Thareja, S., Verma, A., Bhardwaj, T. R., and Kumar, M. (2010). An overview on 5 alpha-reductase inhibitors. Steroids 75, 109-153. doi: 10.1016/j.steroids.2009.10.005

Azzouni, F., Godoy, A., Li, Y., and Mohler, J. (2012). The 5 alpha-reductase isozyme family: a review of basic biology and their role in human diseases. Adv. Urol. 2012:530121. doi: 10.1155/2012/530121

Chaudhary, U. B., and Turner, J. S. (2010). Finasteride. Expert Opin. Drug Metab. Toxicol. 6, 873-881. doi: 10.1517/17425255.2010.495944

Coltman, C. A. Jr., Thompson, I. M. Jr., and Feigl, P. (1999). Prostate cancer prevention trial (PCPT) update. Eur. Urol. 35, 544-547. doi: 10.1159/000019895

Dörsam, J., and Altwein, J. (2009). $5 \alpha$-Reductase inhibitor treatment of prostatic diseases: background and practical implications. Prostate Cancer Prostatic. Dis. 12, 130-136. doi: 10.1038/pcan.2008.56

Guilet, D., Hélesbeux, J.-J., Séraphin, D., Sévenet, T., Richomme, P., and Bruneton, J. (2001). Novel cytotoxic 4-phenylfuranocoumarins from Calophyllum dispar. J. Nat. Prod. 64, 563-568. doi: 10.1021/np000517o

Heinzl, S. (1999). Androgenetic alopecia: finasteride treated hair loss. Med. Monatsschr. Pharm. 22, 124-127.

Kaweetripob, W., Mahidol, C., Prawat, H., and Ruchirawat, S. (2000). Chemical investigation of Mammea siamensis. Pharm. Biol. 38, 55-57. doi: 10.1076/phbi.38.6.55.5954

Kobayashi, M., Akaki, J., Yamaguchi, Y., Yamasaki, H., Ninomiya, K., Pongpiriyadacha, Y., et al. (2019). Salacia chinensis stem extract and its thiosugar sulfonium constituent, neokotalanol, improves HbAlc levels in ob/ob mice. J. Nat. Med. 73, 584-588. doi: 10.1007/s11418-019-01311-w

Koseki, J., Matsumoto, T., Matsubara, Y., Tsuchiya, K., Mizuhara, Y., Sekiguchi, K., et al. (2015). Inhibition of rat $5 \alpha$-reductase activity and testosteroneinduced sebum synthesis in hamster sebocytes by an extract of Quercus acutissima cortex. Evid. Based Complement. Alternat. Med. 2015:853846. doi: $10.1155 / 2015 / 853846$

Laphookhieo, S., Maneerat, W., and Kiattansakul, R. (2006). Phenolic compounds from Mammea siamensis seeds. Can. J. Chem. 84, 1546-1549. doi: 10.1139/v06-157

Laphookhieo, S., Promnart, P., Syers, J. K., Kanjana-Opas, A., Ponglimanont, C., and Karalai, C. (2007). Coumarins and xanthones from the seeds of Mammea siamensis. J. Braz. Chem. Soc. 18, 1077-1080. doi: 10.1590/S0103-50532007000500031

Lee, S. H., Lee, D.-H., Lee, J., Lee, W.-Y., Chung, B. C., and Choi, M. H. (2012). Comparative GC-MS based in vitro assays of $5 \alpha$-reductase activity using rat liver S9 fraction. Mass Spectrom. Lett. 3, 21-24. doi: 10.5478/MSL.2012.3.1.021

Mahidol, C., Kaweetripob, W., Prawat, H., and Ruchirawat, S. (2002). Mammea coumarins from the flowers of Mammea siamensis. J. Nat. Prod. 65, 757-760. doi: 10.1021/np010579u

Mahidol, C., Prawat, H., Kaweetripob, W., and Ruchirawat, S. (2007). Regioisomers of acylcoumarins from the flowers of Mammea siamensis. Nat. Prod. Commun. 2, 557-564. doi: 10.1177/1934578X0700200509

Manse, Y., Ninomiya, K., Nishi, R., Hashimoto, Y., Chaipech, S., Muraoka, O., et al. (2017). Labdane-type diterpenes, galangalditerpenes A-C, with melanogenesis inhibitory activity from the fruit of Alpinia galanga. Molecules 22:2279. doi: $10.3390 /$ molecules 22122279

\section{SUPPLEMENTARY MATERIAL}

The Supplementary Material for this article can be found online at: https://www.frontiersin.org/articles/10.3389/fchem. 2020.00199/full\#supplementary-material

These data include HPLC chromatograms of mammeasins E (2a, 2b) and $\mathrm{F}(\mathbf{3 a}, \mathbf{3 b})$ (Figure S1) ${ }^{1} \mathrm{H}-{ }^{1} \mathrm{H}$ COSY and HMBC correlations of 2 and 3 (Figure S2), 1D and 2D NMR spectra of 1-3 (Figures S3-S17), and inhibitory effects of coumarin constituents (1-35) from $M$. siamensis on testosterone $5 \alpha-$ reductase (Table $\mathbf{S 1}$ ).

Matsuda, H., Sato, N., Yamazaki, M., Naruto, S., and Kubo, M. (2001). Tastosterone $5 \alpha$-reductase inhibitory active constituents from Anemarrhenae Rhizoma. Biol. Pharm. Bull 24, 586-587. doi: 10.1248/bpb.24.586

Morel, C., Guilet, D., Oger, J.-M., Séraphin, D., Sévenet, T., Wiart, C., et al. (1999). 6-Acylcoumarins from Mesua racemose. Phytochemistry 50, 1243-1247. doi: 10.1016/S0031-9422(98)00688-8

Morikawa, T., Manse, Y., Koda, M., Chaipech, S., Pongpiriyadacha, Y., Muraoka, O., et al. (2018). Two new aromatic glycosides, elengiosides A and B, from the flowers of Mimusops elengi. J. Nat. Med. 72, 542-550. doi: 10.1007/s11418-017-1160-z

Morikawa, T., Sueyoshi, M., Chaipech, S., Matsuda, H., Nomura, Y., Yabe, M., et al. (2012). Suppressive effects of coumarins from Mammea siamensis on inducible nitric oxide synthase expression in RAW264.7 cells. Bioorg. Med. Chem. 20, 4968-4977. doi: 10.1016/j.bmc.2012.06.031

Ngo, N. T. N., Nguyen, V. T., Vo, H. V., Vang, O., Duus, F., and Ho, T.-D. H. (2010). Cytotoxic coumarins from the bark of Mammea siamensis. Chem. Pharm. Bull. 58, 1487-1491. doi: 10.1248/cpb.58.1487

Ninomiya, K., Shibatani, K., Sueyoshi, M., Chaipech, S., Pongpiriyadacha, Y., Hayakawa, T., et al. (2016). Aromatase inhibitory activity of geranylated coumarins, mammeasins $\mathrm{C}$ and $\mathrm{D}$, isolated from the flowers of Mammea siamensis. Chem. Pharm. Bull. 64, 880--885. doi: 10.1248/cpb.c16-00218

Noysang, C., Mahringer, A., Zeino, M., Saeed, M., Luanratana, O., Fricker, G., et al. (2014). Cytotoxicity and inhibition of P-glycoprotein by selected medicinal plants from Thailand. J. Ethnopharmacol. 155, 633-641. doi: 10.1016/j.jep.2014.06.001

Petel, D. M., Sharma, M. G., Vala, R. M., Lagunes, I., Puerta, A., Padrón, J. M., et al. (2019). Hydroxyl alkyl ammonium ionic liquid assisted green and one-pot regioselective access to functionalized pyrazolodihydropyridine core and their pharmacological evaluation. Bioorg. Chem. 86, 137-150. doi: 10.1016/j.bioorg.2019.01.029

Poobrasert, O., Constant, H. L., Beecher, C. W. W., Farnsworth, N. R., Kinghorn, A. D., Pezzuto, J. M., et al. (1998). Xanthones from the twigs of Mammea siamensis. Phytochemistry 47, 1661-1663. doi: 10.1016/S0031-9422(97)00820-0

Prachyawarakorn, V., Mahidol, C., and Ruchirawat, S. (2000). NMR study of seven coumarins from Mammea siamensis. Pharm Biol. 38, 58-62. doi: 10.1076/phbi.38.6.58.5962

Prachyawarakorn, V., Mahidol, C., and Ruchirawat, S. (2006a). Siamenols A-D, four new coumarins from Mammea siamensis. Chem. Pharm. Bull 54, 884-886. doi: $10.1248 / \mathrm{cpb} .54 .884$

Prachyawarakorn, V., Mahidol, C., and Ruchirawat, S. (2006b). Pyranocoumarins from the twigs of Mammea siamensis. Phytochemistry 67, 924-928. doi: 10.1016/j.phytochem.2006.02.006

Sangkaruk, R., Rungrojsakul, M., Tima, S., and Anuchapreeda, S. (2017). Effect of Thai saraphi flower extracts on WT1 and Bcr/Abl protein expression in leukemic cell lines. Afr. J. Tradit. Complement. Altern. Med. 14, 16-24. doi: 10.21010/ajtcam.v14i2.3

Sharma, M. G., Vala, R. M., Patel, D. M., Lagunes, I., Fernandes, M. X., Padrón, J. M., et al. (2018). Anti-proliferative 1,4-dihydropyridine and pyridine derivatives synthesized through a catalyst-free, one-pot multi-component reaction. ChemistrySelect 3, 12163-12168. doi: 10.1002/slct.201802537

Tanabe, G., Manse, Y., Ogawa, T., Sonoda, N., Marumoto, S., Ishikawa, F., et al. (2018). Total synthesis of $\gamma$-alkylidenebutenolides, potent melanogenesis 
inhibitors from Thai medicinal plant Melodorum fruticosum. J. Org. Chem. 83, 8250-8264. doi: 10.1021/acs.joc.8b00986

Tanabe, G., Tsutsui, N., Shibatani, K., Marumoto, S., Ishikawa, F., Ninomiya, K., et al. (2017). Total synthesis of the aromatase inhibitors, mammeasins $C$ and D, from Thai medicinal plant Mammea siamensis. Tetrahedron 73, 4481-4486. doi: 10.1016/j.tet.2017.06.016

Titus, M. A., Li, Y., Kozyreva, O. G., Maher, V., Godoy, A., Smith, G. J., et al. (2014). $5 \alpha$-Reductase type 3 enzyme in benign and malignant prostate. Prostate 74, 235-249. doi: 10.1002/pros.22745

Tosti, A., and Piraccini, B. M. (2000). Finasteride and hair cycle. J. Am. Acad. Dermatol. 42, 848-849. doi: 10.1067/mjd.2000.103272

Tung, N. H., Uto, T., Sakamoto, A., Hayashida, Y., Hidaka, Y., Morinaga, O., et al. (2013). Antiproliferative and apoptotic effects of compounds from the flower of Mammea siamensis (Miq.) T Anders. on human cancer cell lines. Bioorg. Med. Chem. Lett. 23, 158-162. doi: 10.1016/j.bmcl.2012.10.127

Uto, T., Tung, N. H., Thongjankaew, P., Lhieochaiphant, S., and Shoyama, Y. (2016). Kayeassamin a isolated from the flower of Mammea siamensis triggers apoptosis by activation caspase-3/-8 in HL-60 human leukemia cells. Pharmacog. Res. 8, 244-248. doi: 10.4103/0974-8490. 188884

Verotta, L., Lovaglio, E., Vidari, G., Finzi, P. V., Neri, M. G., Raimondi, A., et al. (2004). 4-Alkyl- and 4-phenylcoumarins from Mesua ferrea as promising multidrug resistant antibacterials. Phytochemistry 65, 2867-2879. doi: 10.1016/j.phytochem.2004.07.001

Win, N. N., Awale, S., Esumi, H., Tezuka, Y., and Kadota, S. (2008a). Novel anticancer agents, kayeassamins $\mathrm{A}$ and $\mathrm{B}$ from the flower of Kayea assamica of Myanmar. Bioorg. Med. Chem. Lett. 18, 4688-4691. doi: 10.1016/j.bmcl.2008.07.001

Win, N. N., Awale, S., Esumi, H., Tezuka, Y., and Kadota, S. (2008b). Novel anticancer agents, kayeassamins C-I from the flower of Kayea assamica of Myanmar. Bioorg. Med. Chem. 16, 8653-8660. doi: 10.1016/j.bmc.2008. 07.091

Wu, C., and Kapoor, A. (2013). Dutasteride for the treatment of benign prostatic hyperplasma. Expert Opin. Phamacother. 14, 1399-1408. doi: $10.1517 / 14656566.2013 .797965$

Yagi, N., Ohkubo, K., Okuno, Y., Oda, Y., and Miyazawa, M. (2006). Antimutagenic compound from yellow batai (Peltophorum dasyrachis). J. Oleo Sci. 55, 173-180. doi: 10.5650/jos.55.173

Yamana, K., Labrie, F., and Luu-The, V. (2010). Human type $35 \alpha$-reductase is expressed in peripheral tissues at higher levels that types 1 and 2 and its activity is potently inhibited by finasteride and dutasteride. Horm. Mol. Biol. Clin. Invest. 2, 293-299. doi: 10.1515/hmbci.2010.035

Yang, H., Protiva, P., Jiang, B., Baggett, S., Reynertson, K. A., Weinstein, I. B., et al. (2005). Antioxidant and cytotoxic isoprenylated coumarins from Mammea Americana. Planta Med. 71, 852-860. doi: 10.1055/s-2005-871257

Yao, Z., Xu, Y., Zhang, M., Jiang, S., Nicklaus, M. C., and Liao, C. (2011). Discovery of a novel hybrid from finasteride and epristeride as $5 \alpha$-reductase inhibitor. Bioorg. Med. Chem. Lett. 21, 475-478. doi: 10.1016/j.bmcl.2010.10.112

Conflict of Interest: The authors declare that the research was conducted in the absence of any commercial or financial relationships that could be construed as a potential conflict of interest.

Copyright (ㅇ 2020 Morikawa, Luo, Manse, Sugita, Saeki, Chaipech, Pongpiriyadacha, Muraoka and Ninomiya. This is an open-access article distributed under the terms of the Creative Commons Attribution License (CC BY). The use, distribution or reproduction in other forums is permitted, provided the original author(s) and the copyright owner(s) are credited and that the original publication in this journal is cited, in accordance with accepted academic practice. No use, distribution or reproduction is permitted which does not comply with these terms. 